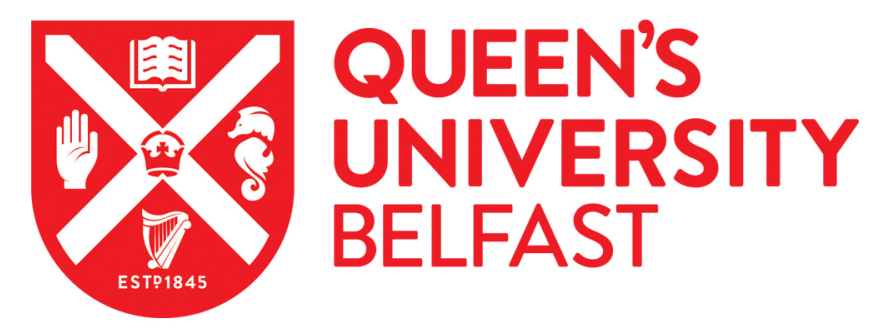

\title{
Cycloidal magnetic order in the compound IrMnSi
}

Eriksson, T., Bergqvist, L., Burkert, T., Felton, S., Tellgren, R., Nordblad, P., Eriksson, O., \& Andersson, Y. (2005). Cycloidal magnetic order in the compound IrMnSi. Physical Review B (Condensed Matter), 71(17), [174420]. https://doi.org/10.1103/PhysRevB.71.174420

\section{Published in:}

Physical Review B (Condensed Matter)

Document Version:

Publisher's PDF, also known as Version of record

Queen's University Belfast - Research Portal:

Link to publication record in Queen's University Belfast Research Portal

Publisher rights

(C) 2005 The American Physical Society

\section{General rights}

Copyright for the publications made accessible via the Queen's University Belfast Research Portal is retained by the author(s) and / or other copyright owners and it is a condition of accessing these publications that users recognise and abide by the legal requirements associated with these rights.

Take down policy

The Research Portal is Queen's institutional repository that provides access to Queen's research output. Every effort has been made to ensure that content in the Research Portal does not infringe any person's rights, or applicable UK laws. If you discover content in the Research Portal that you believe breaches copyright or violates any law, please contact openaccess@qub.ac.uk. 


\title{
Cycloidal magnetic order in the compound IrMnSi
}

\author{
T. Eriksson, ${ }^{1}$ L. Bergqvist, ${ }^{2}$ T. Burkert, ${ }^{2}$ S. Felton, ${ }^{3}$ R. Tellgren, ${ }^{1}$ P. Nordblad,${ }^{3}$ O. Eriksson, ${ }^{2}$ and Y. Andersson ${ }^{1}$ \\ ${ }^{1}$ Department of Materials Chemistry, Uppsala University, Box 538, SE-751 21 Uppsala, Sweden \\ ${ }^{2}$ Department of Physics, Uppsala University, Box 530, SE-751 21 Uppsala, Sweden \\ ${ }^{3}$ Department of Engineering Sciences, Uppsala University, Box 534, SE-751 21 Uppsala, Sweden
}

(Received 7 December 2004; published 24 May 2005)

\begin{abstract}
A new compound, IrMnSi, has been synthesized, and its crystal structure and magnetic properties have been investigated by means of neutron powder diffraction, magnetization measurements, and first-principles theory. The crystal structure is found to be of the TiNiSi type (ordered $\mathrm{Co}_{2} \mathrm{P}$, space group Pnma). The Mn-projected electronic states are situated at the Fermi level, giving rise to metallic binding, whereas a certain degree of covalent character is observed for the chemical bond between the Ir and $\mathrm{Si}$ atoms. A cycloidal, i.e., noncollinear, magnetic structure was observed below $460 \mathrm{~K}$, with the propagation vector $\mathbf{q}=[0,0,0.4530(5)]$ at $10 \mathrm{~K}$. The magnetism is dominated by large moments on the $\mathrm{Mn}$ sites, $3.8 \mu_{B} /$ atom from neutron diffraction. First-principles theory reproduces the propagation vector of the experimental magnetic structure as well as the angles between the Mn moments. The calculations further result in a magnetic moment of $3.2 \mu_{B}$ for the Mn atoms, whereas the Ir and Si moments are negligible, in agreement with observations. A calculation that more directly incorporates electron-electron interactions improves the agreement between the theoretical and experimental magnetic moments. A band mechanism is suggested to explain the observed magnetic order.
\end{abstract}

DOI: 10.1103/PhysRevB.71.174420

PACS number(s): 75.25.+z, 61.66.-f, 71.20.-b, 75.30.Cr

\section{INTRODUCTION}

It is of general interest to study the magnetic properties of manganese compounds, since these often form complex, noncollinear magnetic structures. Examples of this are $\mathrm{Mn}_{3} \mathrm{Sn}$ and $\alpha$ - and $\gamma-\mathrm{Mn}$, as well as $\mathrm{Mn}$ in surface and multilayer geometries. ${ }^{1}$ Several mechanisms have been suggested to be responsible for this peculiar magnetic ordering, e.g., a competition between ferromagnetic and antiferromagnetic interactions of nearest and next-nearest exchange interactions, ${ }^{2}$ geometric frustration, ${ }^{3}$ the Ruderman-KittelKasuya-Yosida (RKKY) interaction, ${ }^{4}$ and lately a mechanism based on band filling and nesting. ${ }^{5}$ Different materials can be classified into different groups, where one of the above-mentioned mechanisms dominates.

One example of a complex magnetic structure is provided by the $\mathrm{Mn}_{3} \mathrm{IrSi}_{1-x} \mathrm{Ge}_{x}(0 \leqslant x \leqslant 1)$ solid solution. These compounds crystallize in an ordered $\beta$-manganese structure, with Mn moments ordering in a $120^{\circ}$ spin configuration - a result of frustrated antiferromagnetic interactions on a threedimensional network of corner-sharing Mn triangles. ${ }^{6,7}$ Further, $\mathrm{Mn}_{3} \mathrm{Sn}$ forms a noncollinear magnetic structure due to relativistic interactions, ${ }^{3}$ whereas the FeNi Invar alloys form noncollinear magnetic structures ${ }^{3}$ according to the mechanism outlined in Ref. 5.

Another example, of direct relevance for this study, is provided by the intermetallic $M M^{\prime} X$ compounds of $1: 1: 1$ stoichiometry (where $M$ and $M^{\prime}$ are transition-metal elements and $X$ is a group 14 or 15 element), which show a variety of interesting magnetic properties. Among the isostructural TiNiSi-type compounds (ordered $\mathrm{Co}_{2} \mathrm{P}$ ), $\mathrm{CoMnGe},{ }^{9} \mathrm{NiMnSi},{ }^{10}$ and $\mathrm{CoMnSi}$ (in the temperature interval $360-390 \mathrm{~K})^{11,12}$ are ferromagnetic and CoMnSi $(T<360 \mathrm{~K})^{11}$ and NiMnGe (Ref. 13) form helicoidal magnetic structures, whereas $\mathrm{NiMnSi}_{0.5} \mathrm{Ge}_{0.5}$ (Ref. 10) and RhMnSi (Ref. 14) form commensurate ferrimagnetic and antiferromagnetic structures, respectively.

The wide range of magnetic properties (ferromagnetism, antiferromagnetism, and noncollinear magnetism) of this class of Mn systems motivates the current combined experimental and theoretical study in the search of different noncollinear magnetic compounds and the identification of the mechanism that stabilizes their magnetic structure. It is of particular interest to contrast the magnetism of the compound we synthesized in this paper, $\mathrm{IrMnSi}$, to the previously discussed $\mathrm{Mn}_{3} \mathrm{IrSi}$, since they are both noncollinear magnets containing $\mathrm{Mn}$, Ir, and $\mathrm{Si}$, but with different stoichiometries and crystal structures. (As we shall see below, IrMnSi crystallizes in a TiNiSi-type structure.)

We determined the crystal and magnetic structures by neutron powder diffraction and also studied the magnetic properties by magnetization measurements. The theoretical total energy and electronic-structure calculations were employed to analyze the origin of the noncollinear order of this compound, using the above-described mechanisms. We came to the conclusion that the band mechanism proposed in Refs. 3 and 5 explains the magnetic order in IrMnSi.

\section{EXPERIMENTAL SECTION}

\section{A. Synthesis}

A sample of stoichiometry IrMnSi was prepared by the drop-synthesis method, ${ }^{15}$ using a high-frequency induction furnace. The sample was contained in an $\mathrm{Al}_{2} \mathrm{O}_{3}$ crucible in a 300-mbar argon atmosphere. The starting materials were pressed pellets of iridium powder (Alfa Aesar, purity 99.95\%), single-crystal pieces of silicon (Highways International, purity 99.999\%), and pieces of manganese 


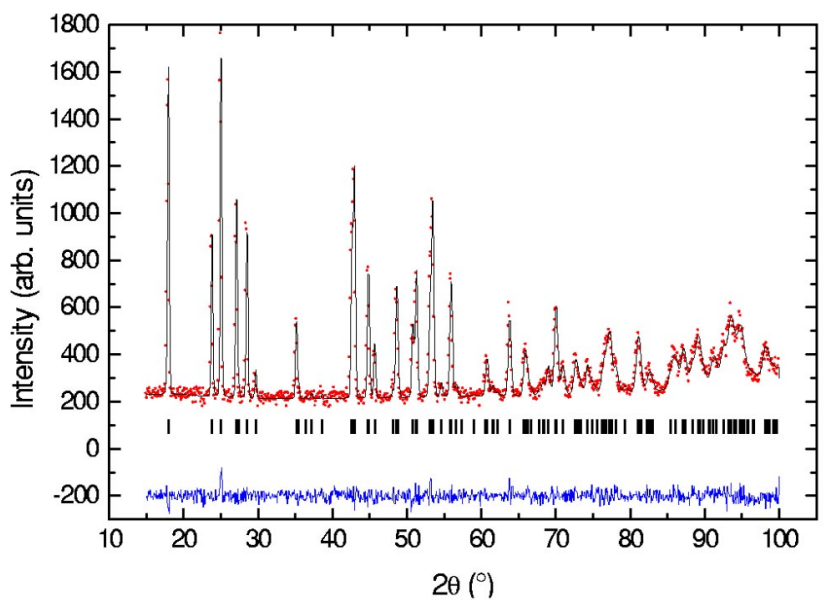

FIG. 1. (Color online) Observed (points), calculated (line), and difference (bottom line) neutron diffraction profiles for IrMnSi at $523 \mathrm{~K}$. The tick marks indicate the positions of the Bragg reflections.

metal (Cerac, claimed purity 99.99\%, purified from manganese oxides by sublimation). The ingot was subsequently crushed and pressed into pellets that were annealed for 4 days at $900{ }^{\circ} \mathrm{C}$ and 8 days at $800{ }^{\circ} \mathrm{C}$ and fine ground and stress relieved at $650{ }^{\circ} \mathrm{C}$ for $40 \mathrm{~min}$ in evacuated silica tubes.

\section{B. X-ray powder diffraction}

Powder x-ray diffraction films were recorded with a Guinier-Hägg focusing camera $\left(\mathrm{Cu} K \alpha_{1}\right.$ radiation) with $\mathrm{Si}$ added as an internal standard [a=5.43088(4) $\AA$ at $295 \mathrm{~K}]$. The sample was found to be single phase with a primitive orthorhombic unit cell of dimensions $a=6.2522(2) \AA$, $b=3.8360(1) \AA, c=7.1255(2) \AA$ at $295 \mathrm{~K}$.

\section{Magnetization measurements}

A Quantum Design MPMS XL superconducting quantum interference device (SQUID) magnetometer was used for the magnetization measurements. The magnetization vs temperature at constant-field sweeps were made from $10 \mathrm{~K}$ to $300 \mathrm{~K}$ in an applied field of 250 Oe using the ordinary cryostat of the system and from $300 \mathrm{~K}$ to $700 \mathrm{~K}$ in an applied field of $1 \mathrm{kOe}$ using the optional oven. The field dependence of the magnetization was recorded at $10 \mathrm{~K}$ and at $700 \mathrm{~K}$. For the temperature-dependent measurements two different protocols were used: zero-field-cooled (ZFC) and field-cooled (FC). In the ZFC protocol the sample is cooled to the starting temperature in zero applied field. Then the desired measurement field is applied and the data are collected on heating the sample. In the FC protocol the measurement field is applied at a temperature above the transition temperature of the sample, and the sample is cooled to the starting temperature in this field. The data are then collected on heating the sample as for the ZFC protocol.

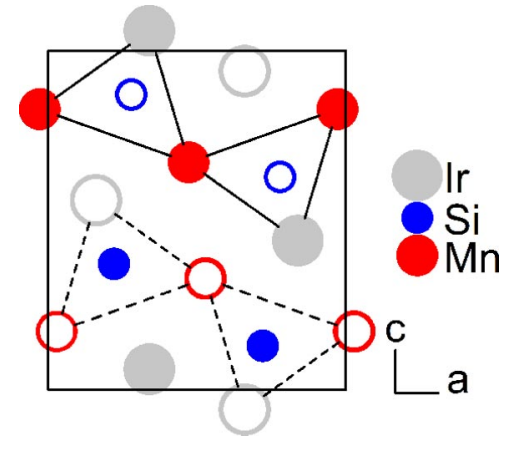

FIG. 2. (Color online) Crystal structure of IrMnSi in projection on the $a c$ plane. Open symbols: $y=\frac{1}{4}$; closed symbols: $y=\frac{3}{4}$.

\section{Neutron powder diffraction}

Neutron powder diffraction intensities were recorded at the Swedish research reactor R2 in Studsvik. The sample was contained in a vanadium cylinder, and the neutron beam was monochromatized by two $\mathrm{Cu}(220)$ single-crystals in a parallel arrangement, giving a wavelength $\lambda=1.471 \AA$. Diffractograms were recorded, with steps of $0.08^{\circ}$, in the $2 \theta$ range $4^{\circ}-139.92^{\circ}$ at the temperatures $523 \mathrm{~K}$ and $295 \mathrm{~K}$, and in the $2 \theta$ range $1.2^{\circ}-139.92^{\circ}$ at $10 \mathrm{~K}$. An absorption correction was applied using $\mu R=1.23$, as calculated from a transmission measurement at $2 \theta=0^{\circ}$.

\section{E. Crystal and magnetic structure refinements}

The structure refinements were performed according to the Rietveld method, using the program FULLPROF. ${ }^{16}$ The neutron-scattering lengths were Ir: $10.6 \mathrm{fm}$; Mn: $-3.73 \mathrm{fm}$; and Si: $4.149 \mathrm{fm}$. The background was modeled by interpolation between fixed points. A pseudo-Voigt profile function with a refined ratio of Gaussian and Lorentzian contributions was used to describe the peak shape.

For the $523 \mathrm{~K}$ data set, 20 parameters were varied. The profile parameters were scale factor (1), zero point (1), profile-shape parameter (1), half-width parameters (3), and asymmetry parameters (2). The structural parameters were atomic coordinates (6), lattice parameters (3), and isotropic temperature factors (3). For the $295 \mathrm{~K}$ and $10 \mathrm{~K}$ data sets, parameters describing the magnetic structure were also varied. A propagation vector $( \pm \boldsymbol{q}$ : 1 parameter varied) was used to describe the magnetic structure. The magnetic moments were localized on the Mn atoms. The real and imaginary components of the magnetic moments were constrained to have the same magnitude $\left(M_{\mathrm{Mn}}\right.$ : 1 parameter varied), with directions described by spherical angles ${ }^{17}(\theta, \varphi, 2$ parameters varied). During refinements of the magnetic structure an overall temperature factor was used, which was fixed to $0.1 \AA^{2}$ and $0.5 \AA^{2}$, respectively, for the $10 \mathrm{~K}$ and $295 \mathrm{~K}$ data sets. The nuclear and magnetic contributions to the diffraction intensities were treated as two separate phases, with the magnetic phase described in space group $P \overline{1}$, using the magnetic form-factor curve of $\mathrm{Mn}^{3+}$.

\section{THEORY}

For the analysis of the chemical bonding a fully relativistic implementation of the full-potential linear muffin-tin or- 
TABLE I. Structural parameters of IrMnSi at $523 \mathrm{~K}$ (estimated standard deviation in parentheses). Space group Pnma (No. 62), Wyckoff position $4 c\left(x, \frac{1}{4}, z\right)$.

\begin{tabular}{llllll}
\hline \hline$a(\AA)$ & $6.2778(6)$ & $b(\AA)$ & $3.8636(4)$ & $c(\AA)$ & $7.1329(6)$ \\
$x_{\mathrm{Mn}}$ & $0.026(1)$ & $x_{\mathrm{Ir}}$ & $0.1598(4)$ & $x_{\mathrm{Si}}$ & $0.7818(9)$ \\
$z_{\mathrm{Mn}}$ & $0.173(1)$ & $z_{\mathrm{Ir}}$ & $0.5602(4)$ & $z_{\mathrm{Si}}$ & $0.6296(9)$ \\
$B_{\mathrm{Mn}}\left(\AA^{2}\right)$ & $1.0(1)$ & $B_{\mathrm{Ir}}\left(\AA^{2}\right)$ & $0.59(5)$ & $B_{\mathrm{Si}}\left(\AA^{2}\right)$ & $0.6(1)$ \\
$R_{\text {profile }}(\%)$ & 4.79 & $R_{w p}(\%)$ & 6.10 & $R_{\exp }(\%)$ & 5.53 \\
$\chi^{2}$ & 1.22 & $R_{\mathrm{Bragg}}(\%)$ & 4.68 & & \\
\hline \hline
\end{tabular}

bital (FP-LMTO) method $^{18}$ was used. In the FP-LMTO method the crystal is divided into nonoverlapping muffin-tin spheres, centered on the atomic sites, and with an interstitial region in between. For the expansion of the electron density and the potential inside the muffin tins, spherical harmonics times a radial component were used. In the interstitial region the expansion made use of a Fourier series. The basis functions were Bloch sums of the Neumann and Hankel functions in the interstitial region that were augmented by a numerical basis function inside the muffin-tin spheres. A so-called double basis was used to ensure a well-converged wave function, i.e., two interstitial basis functions with different tail energies were used, each attached to its own $(n, l)$ radial function. The local spin-density approximation (LSDA) was used for all calculations.

In addition to the type-projected density of states (PDOS) and the charge density, the so-called balanced crystal orbital overlap population ${ }^{19}$ (BCOOP) was used to analyze the character of the chemical bonds. The BCOOP is defined as an orbital-population weighted DOS. It is an energy-resolved quantity which is positive for bonding states and negative for antibonding states, hence enabling a distinction between bonding and antibonding contributions to a chemical bond.

In addition to the FP-LMTO calculations we also performed theoretical calculations based on a noncollinear version of the linear muffin-tin orbital method in the atomic sphere approximation (LMTO-ASA) method $^{20}$ where the atomic magnetic moments can point in a general direction (not simply spin up and spin down) and spiral magnetic structures can be treated. We also performed similar calculations with the projector-augmented wave (PAW) method, ${ }^{21}$ as implemented in Vienna ab-initio simulation package (VASP), where in addition to the LSDA effective potential we also made use of the so-called LSDA $+U$ method, ${ }^{22}$ which takes into account electron-electron interactions in a parametrized Hartree-Fock-like approach.

\section{EXPERIMENTAL RESULTS}

\section{A. Crystal structure}

For the data set recorded at $523 \mathrm{~K}$ the observed intensities are well explained by a TiNiSi-type structure (ordered $\left.\mathrm{Co}_{2} \mathrm{P}\right){ }^{8}$ space group Pnma. Three different $4 c$ positions $\left(x, \frac{1}{4}, z\right)$ are occupied by iridium, manganese, and silicon, respectively, which correspond to four formula units of IrMnSi per unit cell. Within experimental error, all sites are fully occupied, with no evidence of mixed occupancy. In the following magnetic-structure description the Mn atoms will be labeled as follows:

$$
\begin{gathered}
\operatorname{Mn}_{1}\left(x, \frac{1}{4}, z\right), \quad \operatorname{Mn}_{2}\left(x+\frac{1}{2}, \frac{1}{4}, \frac{1}{2}-z\right), \\
\operatorname{Mn}_{3}\left(\frac{1}{2}-x, \frac{3}{4}, \frac{1}{2}+z\right), \quad \operatorname{Mn}_{4}\left(1-x, \frac{3}{4}, 1-z\right) .
\end{gathered}
$$

Structure refinements were performed by the Rietveld method for the $2 \theta$-range $15^{\circ}-100^{\circ}$ due to extensive peak overlap at higher angles, resulting in agreement factors $R_{\text {profile }}=4.79 \%, R_{\text {Bragg }}=4.68 \%$ in the last cycle of refinements. The fitted diffractogram at $523 K$ is plotted in Fig. 1, and the obtained structural parameters are listed in Table I. The same crystal structure is found at $295 \mathrm{~K}$ and $10 \mathrm{~K}$, with only small changes of the atomic coordinates. The interatomic distances shorter than $3 \AA$ at $10 \mathrm{~K}$ were calculated and listed in Table II.

A projection of the IrMnSi structure on the $a c$ plane is shown in Fig. 2. All atoms are situated on mirror planes perpendicular to the $b$ axis, at heights $y=\frac{1}{4}$ and $y=\frac{3}{4}$. The silicon atoms are located in triangular prisms made up of two $\mathrm{Ir}+$ four $\mathrm{Mn}$ atoms, with further two Ir and one Mn atoms (on corners of other prisms) capping the rectangular sides of the prisms on the same height as the Si atom. Prisms on the same

TABLE II. Interatomic distances $(\AA)$ in IrMnSi at $10 \mathrm{~K}$ (estimated standard deviation in parentheses).

\begin{tabular}{llllll}
\hline \hline $\mathrm{Mn}-\mathrm{Si}$ & $2.664(8)$ & $\mathrm{Ir}-\mathrm{Si}$ & $2.335(6)$ & $\mathrm{Si}-\mathrm{Ir}$ & $2.335(6)$ \\
$\mathrm{Mn}-2 \mathrm{Si}$ & $2.674(6)$ & $\mathrm{Ir}-2 \mathrm{Si}$ & $2.372(4)$ & $\mathrm{Si}-2 \mathrm{Ir}$ & $2.372(4)$ \\
$\mathrm{Mn}-2 \mathrm{Si}$ & $2.718(6)$ & $\mathrm{Ir}-\mathrm{Si}$ & $2.424(6)$ & $\mathrm{Si}-\mathrm{Ir}$ & $2.424(6)$ \\
$\mathrm{Mn}-\mathrm{Ir}$ & $2.820(6)$ & $\mathrm{Ir}-\mathrm{Mn}$ & $2.820(6)$ & $\mathrm{Si}-\mathrm{Mn}$ & $2.664(8)$ \\
$\mathrm{Mn}-2 \mathrm{Ir}$ & $2.853(5)$ & $\mathrm{Ir}-2 \mathrm{Mn}$ & $2.853(5)$ & $\mathrm{Si}-2 \mathrm{Mn}$ & $2.674(6)$ \\
$\mathrm{Mn}-\mathrm{Ir}$ & $2.891(6)$ & $\mathrm{Ir}-\mathrm{Mn}$ & $2.891(6)$ & $\mathrm{Si}-2 \mathrm{Mn}$ & $2.718(6)$ \\
$\mathrm{Mn}-2 \mathrm{Ir}$ & $2.940(5)$ & $\mathrm{Ir}-2 \mathrm{Ir}$ & $2.897(3)$ & & \\
& & $\mathrm{Ir}-2 \mathrm{Mn}$ & $2.940(5)$ & & \\
\hline \hline
\end{tabular}




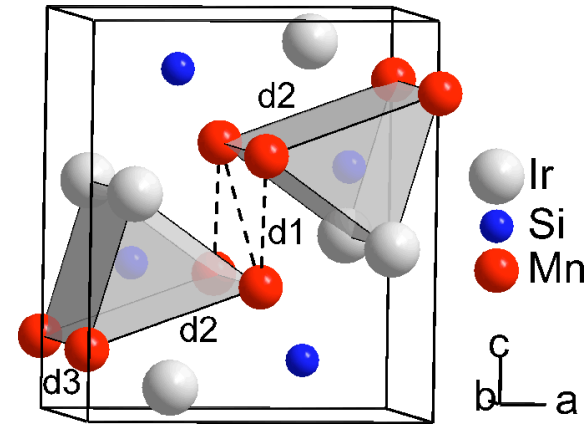

FIG. 3. (Color online) Crystal structure of IrMnSi with shaded prisms around $\mathrm{Si}$ and the three closest $\mathrm{Mn}-\mathrm{Mn}$ distances indicated $\left(d_{1}\right.$, dashed lines between the prisms; $d_{2}, d_{3}$ on the edges of the prisms).

height along the $b$ axis share two corners, forming buckled chains that propagate along the $a$ axis and form infinite columns along the $b$ axis. The local surroundings of the Mn atoms are distorted square pyramids of five $\mathrm{Si}$ atoms, whereas the Ir atoms are located in irregular tetrahedra of four $\mathrm{Si}$ atoms.

To illustrate where magnetic interactions between the $\mathrm{Mn}$ atoms can be expected, the crystal structure is also displayed in Fig. 3, with shaded prisms around two of the Si atoms (at $y=\frac{1}{4}$ and $y=\frac{3}{4}$ ) and with a dashed line between neighboring $\mathrm{Mn}$ atoms on different chains of prisms. The shortest $\mathrm{Mn}-\mathrm{Mn}$ distance is found along the dashed lines between prisms on different heights along the $b$ axis $\left[d_{1}=3.124(9) \AA\right.$ at $\left.10 \mathrm{~K}\right]$. The second-nearest Mn-Mn distance corresponds to the edges of the triangular faces of the prisms $\left[d_{2}=3.313(4) \AA\right.$ at $\left.10 \mathrm{~K}\right]$. The height of the prisms $\left[d_{3}=3.8275(3) \AA\right.$ at $\left.10 \mathrm{~K}\right]$ separate the third-nearest $\mathrm{Mn}$ neighbors.

\section{B. Magnetization measurements}

The results of the magnetization-vs-temperature measurements are shown in Figs. 4 and 5. The susceptibility $(\chi=M / H)$ is paramagnetic and quite weakly temperature dependent. The most prominent feature of the susceptibility-vs-

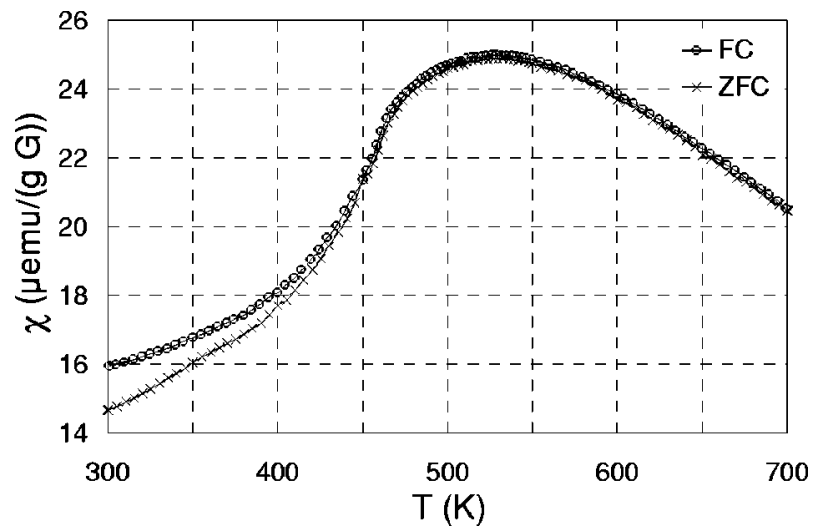

FIG. 4. Magnetic susceptibility $(\chi)$ vs temperature $(T)$, $300-700 \mathrm{~K}$, measured with an applied field of $1 \mathrm{kG}$.

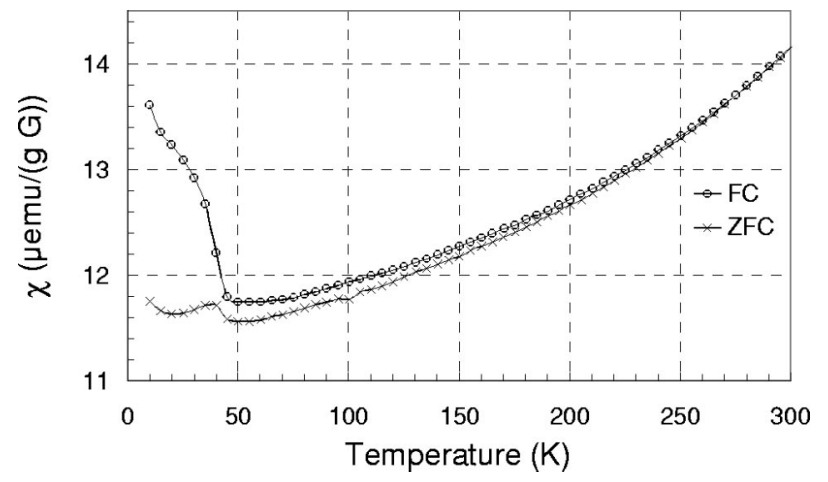

FIG. 5. Magnetic susceptibility $(\chi)$ vs temperature $(T)$, $10-300 \mathrm{~K}$, measured with an applied field of $250 \mathrm{G}$.

temperature curve is a broad maximum at about $570 \mathrm{~K}$ that indicates that the system approaches an "antiferromagnetic" (compensated spontaneous magnetization) phase transition. The transition temperature, derived from the maximum in the $d(\chi T) / d T$-vs- $T$ curve, is found to be $T_{N}=460 \mathrm{~K}$. The very broad maximum and the huge difference between the temperature for the maximum and the transition temperature implies that the magnetic order is not that of an ordinary $3 d$ antiferromagnet. As can be seen from Fig. 4, our experiment did not reach a region where the system shows a CurieWeiss behavior, which hinders an experimental determination of $p_{\text {eff }}$.

An additional feature is observed at about $40 \mathrm{~K}$, where the ZFC and FC magnetization curves suddenly deviate and the FC susceptibility rapidly increases. From the magnetization data it is not possible to distinguish whether this anomaly arises from some very small amount (not resolved in the diffraction measurements) of a ferromagnetic impurity phase with a transition temperature at $40 \mathrm{~K}$ (e.g., $\mathrm{Mn}_{3} \mathrm{O}_{4}$ ) or if it is due to some minor rearrangement of the spin structure that causes a less-well-compensated spontaneous magnetic moment in the sample. The similarity between the magnetic structure at $10 \mathrm{~K}$ and $295 \mathrm{~K}$ from the neutron diffraction does not give any indication of a spin reconfiguration between these temperatures. The magnetization curves at $10 \mathrm{~K}$ and $700 \mathrm{~K}$ yielded a paramagnetic response (linear $M$-vs- $H$ curves) with a susceptibility of $1.2 \times 10^{-5} \mathrm{emu} /(\mathrm{g} \mathrm{Oe})$ at $10 \mathrm{~K}$ and $2.0 \times 10^{-5} \mathrm{emu} /(\mathrm{g} \mathrm{Oe})$ at $700 \mathrm{~K}$. (It should be noted that the high-temperature experiments were made with a sample holder that gives a larger background signal that causes some difference in the absolute value of the measured susceptibility between the data at high and low temperatures.)

\section{Magnetic structure}

In addition to the peaks present at $523 \mathrm{~K}$, the diffractograms recorded at $295 \mathrm{~K}$ and $10 \mathrm{~K}$ (shown in Fig. 6) show reflections that are of magnetic origin. A $(000)^{ \pm}$reflection as well as satellites around the nuclear reflections were successfully indexed by a propagation vector parallel to the $c$ axis, $\mathbf{q}=\left(0,0, q_{z}\right) ; q_{z}=0.4530(5)$ at $10 \mathrm{~K}$ and $q_{z}=0.4541(5)$ at $295 \mathrm{~K}$, corresponding to a repeat distance of $\sim 15.7 \AA$. The 


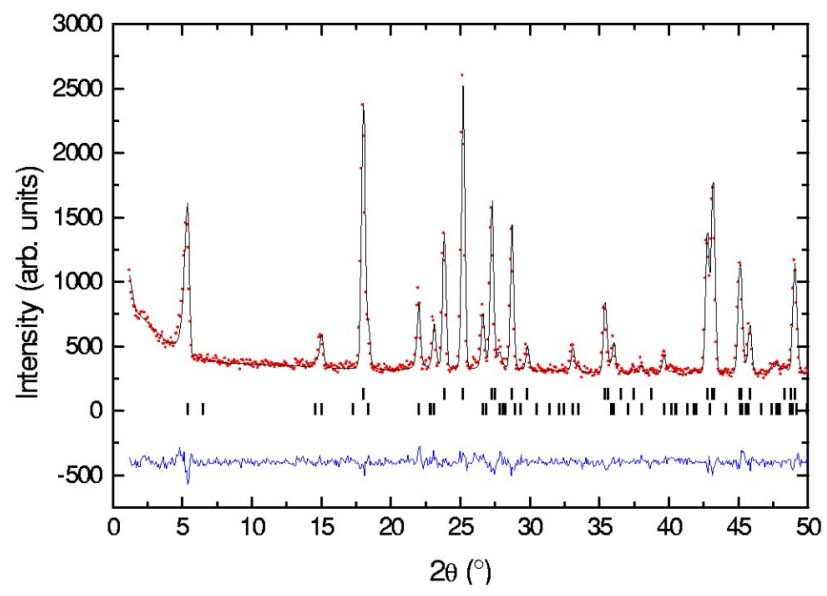

FIG. 6. (Color online) Observed (points), calculated (line), and difference (bottom line) neutron diffraction profiles for IrMnSi at $10 \mathrm{~K}$. The upper and lower tick marks indicate the positions of the Bragg reflections for the crystal and magnetic structures, respectively.

best fit to the observed intensities was at both temperatures obtained for a cycloidal magnetic structure model, with magnetic moments on the Mn atoms. The magnetic moments rotate within a plane comprising the propagation vector, i.e., they rotate around an axis lying in the $a b$ plane, with the angle between the $a$ axis and the plane of rotation varied during the refinements [obtained value $63(3)^{\circ}$ at $10 \mathrm{~K}$, $74(6)^{\circ}$ at $295 \mathrm{~K}$ ]. No magnetic moment on the $\mathrm{Ir}$ sites was detected, within the accuracy of the neutron diffraction experiments.

The magnetic moment $M(L, j)$ on the $\mathrm{Mn}$ atom $j$ in unit cell $L$, related to the reference cell $\left(n_{i}=0\right)$ by the lattice vector $\boldsymbol{R}(L)=\mathrm{n}_{1} \mathbf{a}+\mathrm{n}_{2} \mathbf{b}+\mathrm{n}_{3} \mathbf{c}$ ( $n_{i}=$ integer), is given by

$$
\begin{aligned}
M(L, j)= & \operatorname{Re}(j) \cos \{2 \pi \cdot[\boldsymbol{q} \cdot \boldsymbol{R}(L)+\psi(j)]\} \\
& +\operatorname{Im}(j) \sin \{2 \pi \cdot[\boldsymbol{q} \cdot \boldsymbol{R}(L)+\psi(j)]\},
\end{aligned}
$$

where $\mathbf{q}$ is the propagation vector, $\psi(j)$ is the phase angle (in fractions of $2 \pi), \operatorname{Re}(j)$ and $\operatorname{Im}(j)$ are the real and imaginary Fourier coefficients of the magnetic moment, and

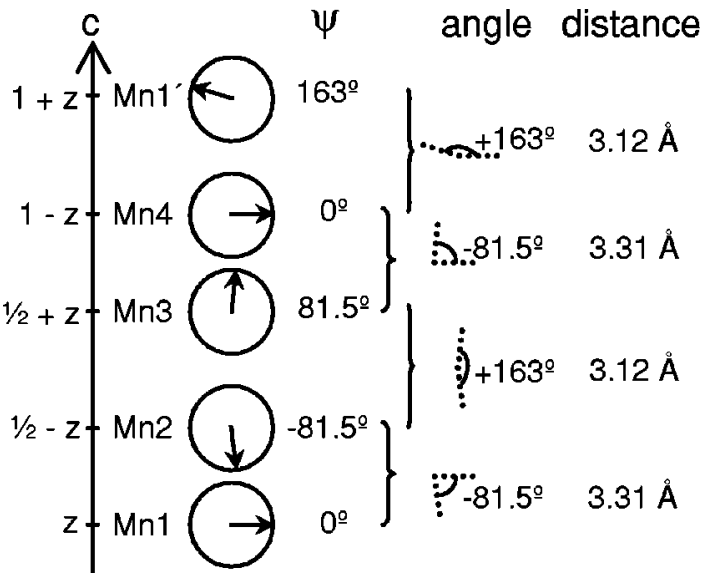

FIG. 7. Magnetic-moment directions (view direction normal to the plane of rotation), with the phase angle $(\psi)$ as well as angles and distances between neighboring $\mathrm{Mn}$ atoms indicated (corresponding to $d_{1}, d_{2}$ in Fig. 3).

$$
M_{\mathrm{Mn}}=|\operatorname{Re}(j)|=|\operatorname{Im}(j)| .
$$

The best fit between the observed and calculated intensities was obtained with the phase angles of the four Mn atoms within one crystallographic unit cell related as (numbered as defined in the crystal structure section):

$$
\psi_{\mathrm{Mn} 1}=\psi_{\mathrm{Mn} 4}=\psi_{1}=0, \quad-\psi_{\mathrm{Mn} 2}=\psi_{\mathrm{Mn} 3}=\psi_{2}=\frac{1}{2} q_{z}
$$

Thus the magnetic moments on $\mathrm{Mn}_{1}$ and $\mathrm{Mn}_{4}$ are parallel, whereas the angle between the magnetic moments of atoms separated by $\frac{1}{2}$ unit cell along the propagation vector $\left(\mathrm{Mn}_{1}-\mathrm{Mn}_{3} ; \mathrm{Mn}_{2}-\mathrm{Mn}_{4}\right)$ is $\frac{1}{2} q_{z} \cdot 360^{\circ} \approx 81.5^{\circ}$.

The Fourier coefficients of the magnetic moments placed on the Mn atoms, resulting from structure refinements for the $10 \mathrm{~K}$ data set, are listed in Table III, together with cell parameters, atom coordinates, propagation vector, phase angles, and agreement factors. The observed and calculated profiles at $10 \mathrm{~K}$ are plotted in Fig. 6 .

With the phase angles fixed during the refinements, the obtained magnitude of the magnetic moment was $3.77(4) \mu_{B}$ at $10 \mathrm{~K}$, with the magnetic agreement factor $R_{\mathrm{Mag}}=8.58 \%$.

TABLE III. Structural and magnetic parameters of IrMnSi at $10 \mathrm{~K}$ (estimated standard deviation in parentheses).

\begin{tabular}{llllll}
\hline \hline$a(\AA)$ & $6.2415(5)$ & $b(\AA)$ & $3.8275(3)$ & $c(\AA)$ & $7.1220(6)$ \\
$x_{\mathrm{Mn}}$ & $0.0258(9)$ & $x_{\mathrm{Ir}}$ & $0.1600(4)$ & $x_{\mathrm{Si}}$ & $0.7795(9)$ \\
$z_{\mathrm{Mn}}$ & $0.1719(8)$ & $z_{\mathrm{Ir}}$ & $0.5604(4)$ & $z_{\mathrm{Si}}$ & $0.6290(8)$ \\
$M_{\mathrm{Mn}}\left(\mu_{B}\right)$ & $3.77(4)$ & $\psi_{1}(2 \pi)^{\mathrm{a}, \mathrm{b}}$ & 0.0 & $\psi_{2}(2 \pi)^{\mathrm{a}}$ & $0.2265(2)$ \\
$\varphi_{\operatorname{Re}}\left({ }^{\circ}\right)$ & $63(3) .0$ & $\theta_{\operatorname{Re}}\left({ }^{\circ}\right)^{\mathrm{b}}$ & 90.0 & $q_{z}$ & $0.4530(5)$ \\
$\varphi_{\mathrm{Im}}\left({ }^{\circ}\right)^{\mathrm{b}}$ & 0.0 & $\theta_{\mathrm{Im}}\left({ }^{\circ}\right)^{\mathrm{b}}$ & 0.0 & $B_{\text {overall }}\left(\AA^{2}\right)^{\mathrm{b}}$ & 0.1 \\
$R_{\text {profile }}(\%)$ & 4.40 & $R_{w p}(\%)$ & 5.56 & $R_{\mathrm{exp}}(\%)$ & 4.62 \\
$\chi^{2}$ & 1.45 & $R_{\mathrm{Bragg}}(\%)$ & 3.53 & $R_{\mathrm{Mag}}(\%)$ & 8.58 \\
\hline \hline
\end{tabular}

aphase angle $\left(\psi_{j}\right): \psi_{\mathrm{Mn} 1}=\psi_{\mathrm{Mn} 4}=\psi_{1} ;-\psi_{\mathrm{Mn} 2}=\psi_{\mathrm{Mn} 3}=\psi_{2}=\frac{1}{2} q_{z}$.

${ }^{b}$ Fixed values.

$\varphi, \theta$ : Spherical angles of the (real and imaginary) Fourier components of the magnetic moment. 


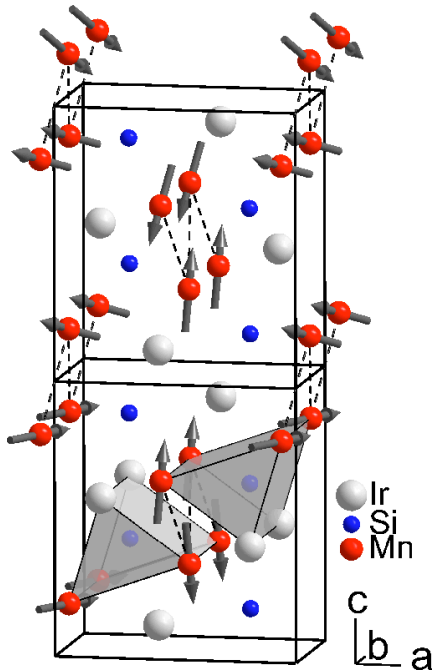

FIG. 8. (Color online) Three-dimensional view of the magnetic moments in consecutive unit cells along the propagation vector (c-axis direction).

Allowing $\psi_{\mathrm{Mn} 4}$ to vary (setting $\psi_{\mathrm{Mn} 2}=\psi_{\mathrm{Mn} 4}-\frac{1}{2} q_{z}$ ) gives a better agreement factor $\left(R_{\mathrm{Mag}}=6.53 \%\right)$, and results in a small negative phase angle $\left[\psi_{\mathrm{Mn} 4}=-0.008(3)\right]$, accompanied by an increase of the magnitude of the magnetic moments to 3.95(6) $\mu_{B}$. However, the difference between the two models is so small that within experimental accuracy the $\mathrm{Mn}_{1}$ and $\mathrm{Mn}_{4}$ moments can be regarded as parallel. As can be expected, a lower magnetic moment is obtained at $295 \mathrm{~K}$ : $M_{\mathrm{Mn}}=3.06(4) \mu_{B}$.

The magnetic-moment directions are drawn in the plane of rotation in Fig. 7, with the angle and distance between the neighboring $\mathrm{Mn}$ moments indicated. A three-dimensional

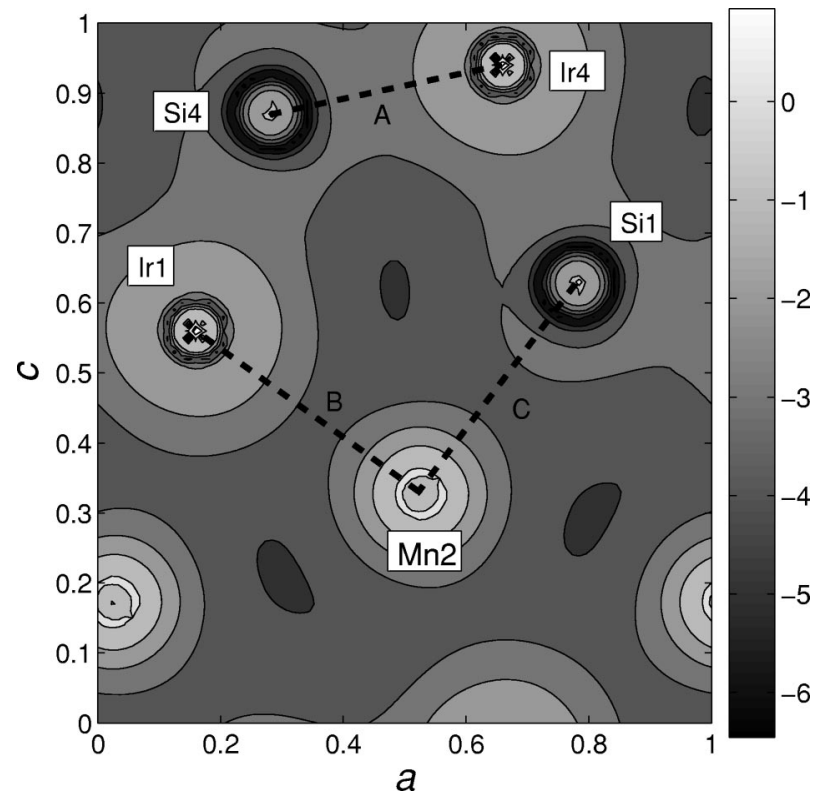

FIG. 9. Logarithmic charge density of IrMnSi projected onto the $a c$ plane at $y=\frac{1}{4}$ (open symbols in Fig. 2). The enumerated dashed lines show the bonds investigated by the BCOOP presented in Fig. 10.
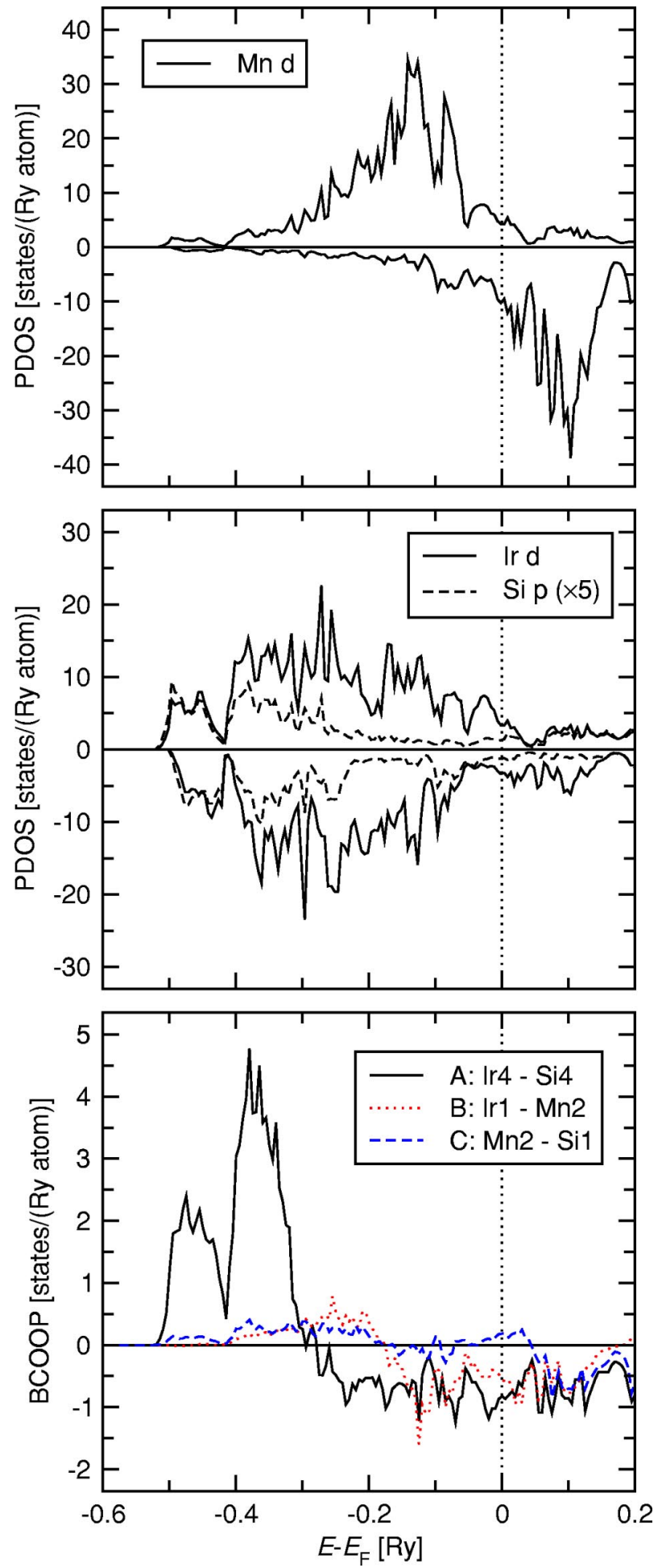

FIG. 10. (Color online) PDOS of Mn $d$ (upper panel) and Ir $d$, and $\mathrm{Si} p$ (middle panel). Note that the Si $p$ PDOS is enlarged by a factor of 5. The lower panel shows the BCOOP of IrMnSi for some selected bonds (cf. Fig. 9).

view of the magnetic moments in consecutive unit cells along the propagation vector (c-axis direction) were calculated from Eq. (1) and are illustrated in Fig. 8. Within the first unit cell the directions are given by $\psi_{\mathrm{Mn} j} \cdot 360^{\circ}$, with all directions further rotated 
$n q_{z} \cdot 360^{\circ} \approx n \cdot 163^{\circ}$ in the following $(n=1,2, \ldots)$ unit cells. This renders the magnetic moments on pairs of nearneighbor $\mathrm{Mn}$ atoms (separation distances $d_{1}$ and $d_{2}$, respectively) to have $163^{\circ}$ and $82^{\circ}$ angles between them, respectively, due to their different intersheet distances along the propagation vector (see Figs. 7 and 8).

\section{THEORETICAL RESULTS}

\section{A. Crystal chemistry}

In Fig. 9 the charge-density distribution of $\mathrm{IrMnSi}$ is shown projected onto the $a c$ plane at $y=\frac{1}{4}$ (cf. Fig. 2). Note that the charge density of the $y=\frac{3}{4}$ plane is similar to that of Fig. 9, but it has the mirror image of Fig. 9. The charge density around the Mn atoms is spherical with a region outside the atoms that is rather featureless and flat, which points towards a mainly metallic bond. Along the bonds between the Ir and Si atoms, on the other hand, some charge is accumulated, which is characteristic of a covalent character to the bond. It should be noted that there is still an overall metallic component to the chemical bond of this material, and in fact the electronic structure has bands crossing the Fermi level $\left(E_{F}\right)$. This is reflected in the density of states (DOS), shown in Fig. 10.

In order to further analyze the bonding character, the DOS projected on the atomic types, as well as the BCOOP calculated for some selected bonds, are plotted in Fig. 10. Comparing the Mn $d$ PDOS (upper panel in Fig. 10) with those of Ir $d$ and Si $p$ (middle panel) supports the conclusions drawn from the charge-density distribution-the very similar PDOS of the Ir $d$ states and the Si $p$ states are characteristic of strong hybridization and, as a result, a covalent bond. The Mn $d$ PDOS, on the other hand, shares no common features with those of either Ir or $\mathrm{Si}$, which points towards a much smaller hybridization and a less covalent bonding, which is consistent with the metallic bond discussed in connection with Fig. 9.

The BCOOP of selected Ir-Mn, Si-Mn, and $\mathrm{Si}-\mathrm{Ir}$ bonds is plotted in the lower panel of Fig. 10. For a pure covalent bond the $\mathrm{BCOOP}$ has positive and negative regions that are well separated. If $E_{F}$ cuts through a region that separates the positive region of the BCOOP curve from the negative region, a maximum of the covalent binding is expected. ${ }^{19}$ The $\mathrm{BCOOP}$ between the Ir and Si pairs shows a positive region in the energy range of -0.5 to $-0.3 \mathrm{Ry}$ (indicating bonding covalent states) and negative contributions in the range between -0.3 Ry and the Fermi energy (indicating antibonding states). It should be noted that all bonding states are filled, whereas only a part of the antibonding states are filled, resulting in a net covalent contribution to the chemical bonds. In Fig. 10 we also note that the BCOOP curve for the $\mathrm{Mn}$-Ir pair and the $\mathrm{Mn}$-Si pair signals to a much lesser degree a distinct division of states into bonding and antibonding types, which suggests that the covalence between these atom types is much less pronounced, compared to the $\mathrm{Si}$-Ir bond. This conclusion is consistent with the discussion around Fig. 9.

\section{B. Magnetic structure}

The magnetic properties were calculated both in the LMTO-ASA formalism, from the FP-LMTO technique as

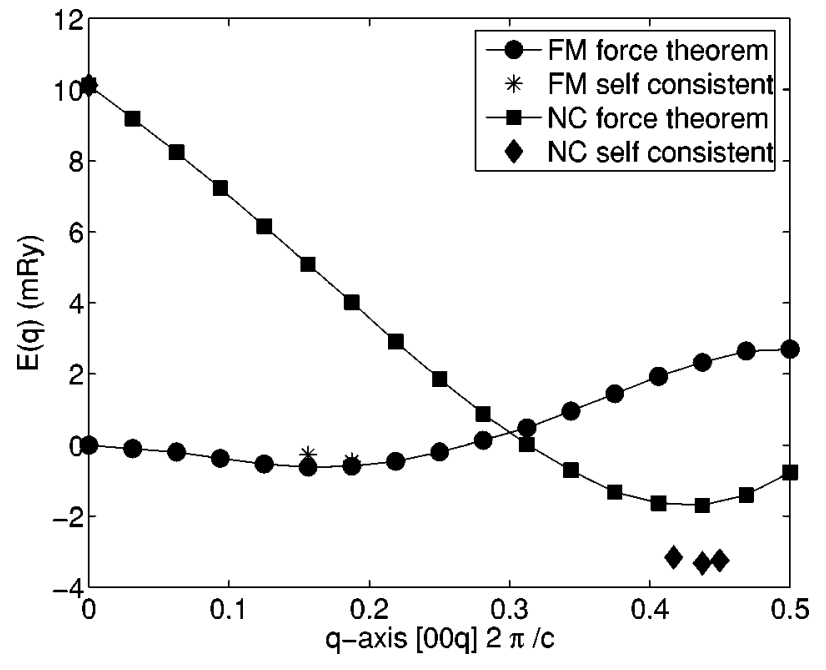

FIG. 11. Calculated energy difference (per formula unit) between the ferromagnetic configuration and a noncollinear, screw-spiral configuration. The reference level is the ferromagnet, which defines the energy zero in the plot. FM denotes a ferromagnetic coupling between the four $\mathrm{Mn}$ atoms inside the crystallographic unit cell, and NC denotes angles between the Mn atoms inside the crystallographic cell that equal the experimental values.

well as by using the PAW method as implemented in VASP. For the latter method we also compared results from the LSDA with calculations using the LSDA $+U$ approximation. A ferromagnetic calculation based on the LSDA results in a magnetic moment just above $3 \mu_{B} / \mathrm{Mn}$ atom (3.1 with LMTO-ASA, 3.3 with FP-LMTO, and 3.3 with VASP), with an almost-vanishing moment on all other atoms of the cell. This deviates to some extent from the experimental values, where the magnetism is indeed found to reside on the $\mathrm{Mn}$ atoms, but with a larger magnitude of $3.8 \mu_{B} / \mathrm{Mn}$ atom.

We also investigated the energy of different magnetic phases in this compound. In Fig. 11 we show the energy of a ferromagnetic alignment of the four Mn atoms inside the crystallographic unit cell, but with a screw-spiral geometry with a propagation vector $(\mathbf{q})$ along the [001] direction. It is to be noted that an energy minimum is observed at $q \sim 0.17$. We also investigated the energy of the experimental magnetic structure. This was done by fixing the angles of the magnetic moments of the four $\mathrm{Mn}$ atoms inside a given unit cell to the experimental values, ${ }^{23}$ and then the total energy was calculated for different values of $\mathbf{q}$. The results are shown in Fig. 11, and we observe that the energy minimum is found at $q \sim 0.43$, which is in good agreement with our experimental values.

The theoretical results so far have been extracted by means of the force theorem, which is a computationally efficient method of comparing energy differences between magnetic structures, at the expense of being slightly less accurate than a comparison of total energies of fully selfconsistent calculations. In order to assess the accuracy of the force theorem, we also performed fully self-consistent calculations, and the energies of these calculations are also shown in Fig. 11. Note from the figure that for the fully self- 


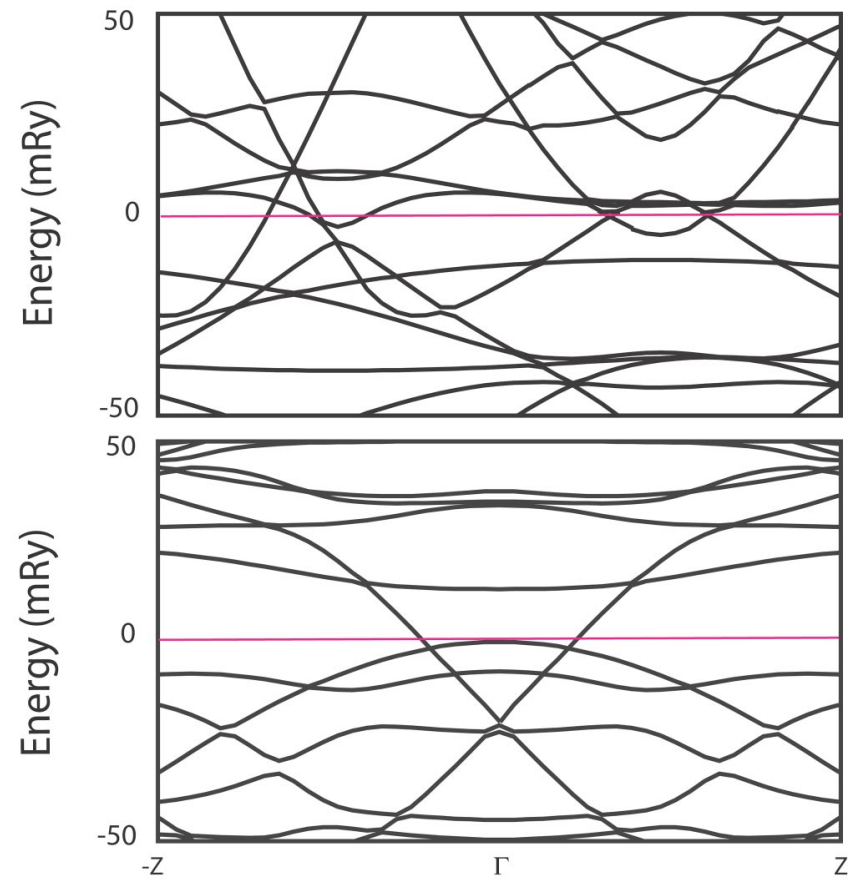

FIG. 12. (Color online) Calculated energy bands for IrMnSi. The Fermi level is at zero and is marked by a thin horizontal line. In the upper panel the bands for a ferromagnetic alignment of the four Mn spins within the crystallographic cell are displayed. In the lower panel the band structure of IrMnSi for the experimental configuration of the Mn spins is shown.

consistent calculations the energy minimum is moved down in energy with 1-2 $\mathrm{mRy} /$ formula unit, but the $q$ value for this minimum is more or less unchanged. For the selfconsistent calculations we also minimized the energy with respect to the angles of the $\mathrm{Mn}$ atoms inside the crystallographic unit cell, but the deviation from the experimental values was very small ( $\sim 3^{\circ}$ on average). The self-consistent calculations for this noncollinear magnetic structure resulted in somewhat larger Mn moments compared to the ferromagnetic calculations, but a value of $3.2 \mu_{B} / \mathrm{Mn}$ atom is still $\sim 15 \%$ lower than the observed value. From Fig. 11 it is now obvious that one observes an energy minimum for a magnetic structure that is very close to the experimental one (the $q$ value is almost identical as are the angles between the magnetic atoms inside the crystallographic unit cell). Both in the fully self-consistent calculations and the calculations based on the force theorem this minimum is just barely an absolute minimum.

In order to analyze the origin of the complex magnetic order in IrMnSi we show in Fig. 12 the energy-band structure along the $-\mathrm{Z}-\Gamma-\mathrm{Z}$ direction. In the top part we show the energy bands corresponding to a wave function with a $q$ value equal to the experimental one. ${ }^{3}$ This wave function is characterized by the $q$ vector and the angle $\theta \cdot{ }^{3,20}$ The value of $\theta$ in this calculation was set to zero, which corresponds to a ferromagnetic arrangement of the spins and also a ferromagnetic total energy. The upper panel of Fig. 12 is hence nothing but a complex way to plot the energy bands of a ferromagnet, using a general wave function with spin-spiral symmetry. ${ }^{3}$ In the lower panel of
Fig. 12 we show the energy bands of IrMnSi for the observed magnetic structure, i.e., for a wave function with the experimental $q$ value and using $\theta=90 .{ }^{23}$ In the past a mechanism has been identified that stabilizes the energy of noncollinear magnets and is based on an energy-band analysis., ${ }^{3,5}$ For the noncollinear magnetic order, the bands start to hybridize with one another, especially bands that for a ferromagnet are orthogonal (i.e., spin-up and spin-down bands of the ferromagnet). If this causes an opening of the band gaps around $E_{F}$, so that the number of bands that cross $E_{F}$ is reduced, the total energy of the system is lowered. ${ }^{3,5}$ When comparing the two panels of Fig. 12 we observe that this indeed is the case, and we conclude that IrMnSi belongs to the class of materials that are stabilized due to the band mechanism of Refs. 3 and 5.

In the past it has been found that the so-called $\mathrm{LSDA}+U$ approximation sometimes gives larger magnetic moments, and for this reason we also performed calculations using this technique. For simplicity this was done for a ferromagnetic configuration. Values of $U$ of 2,3 , and $4 \mathrm{eV}$ for the $\mathrm{Mn}$ atom were chosen, and for the other atoms $U$ was zero. As expected one indeed obtains larger values for the Mn moments; for a $U$ of $2 \mathrm{eV}$ we calculate a Mn moment of $3.7 \mu_{B}$ /atom, $U=3 \mathrm{eV}$ results in a Mn moment of $3.9 \mu_{B} / \mathrm{Mn}$ atom, and finally $U=4 \mathrm{eV}$ yields a $\mathrm{Mn}$ moment of $4.1 \mu_{B} / \mathrm{Mn}$ atom. This result is quite encouraging since these values of $U$ have been discussed for metallic $3 d$ elements. $^{24}$

In Table IV we compare the calculated structural parameters as a function of selected values of $U$ (in the range of $2-4 \mathrm{eV}$ ). The TiNiSi structure has several free parameters; the lattice parameter $c / a$ and $b / a$ ratios, as well as positional parameters for all the atoms. It may be noted that as a function of $U$ the calculated structural result is approaching the experimental values, and that the calculation with $U=4$ comes closest to the measured data. Hence we conclude that both the magnetic moments and structural properties are best reproduced with the LSDA $+U$ approach using a $U$ of $4 \mathrm{eV}$.

\section{DISCUSSION AND CONCLUSIONS}

IrMnSi was found to crystallize with the TiNiSi type structure, and it is thus isostructural with the roomtemperature polymorph of, e.g., $\mathrm{RhMnSi}^{14}{ }^{14} \mathrm{CoMnSi},{ }^{11,12}$ CoMnGe, ${ }^{9} \mathrm{NiMnSi},{ }^{10}$ and NiMnGe. ${ }^{13,25}$ For $\mathrm{CoMn}(\mathrm{Si} / \mathrm{Ge})$ and $\mathrm{NiMn}(\mathrm{Si} / \mathrm{Ge})$ a transition to the $\mathrm{Ni}_{2}$ In-type structure is reported to take place at higher temperatures (between 125 and $\left.930{ }^{\circ} \mathrm{C}\right) .{ }^{26}$ However, we find no traces of a $\mathrm{Ni}_{2}$ In-type phase in the IrMnSi samples quenched from $800{ }^{\circ} \mathrm{C}$, nor do we see any evidence for such a structural transition on heating to $523 \mathrm{~K}$. As in the above-listed compounds, Mn occupies the pyramidal site in IrMnSi. This is different from the TiNiSi-type $R \mathrm{MnSi}$ and $R \mathrm{MnGe}$ compounds $(R=$ rare earth element), where $R$ occupies the pyramidal site and Mn the tetrahedral position. ${ }^{27-29}$

In addition to an overall metallic binding in $\operatorname{IrMnSi}$, a certain degree of a covalent bond character is observed between Ir and Si in the theoretically calculated charge-density 
TABLE IV. Comparison between structural parameters calculated by LSDA, LSDA $+U(U=2-4)$, and the experimentally obtained parameters (at $10 \mathrm{~K}$ ).

\begin{tabular}{|c|c|c|c|c|c|c|c|c|c|}
\hline & Vol. $\left(\AA^{3}\right)$ & $b / a$ & $c / a$ & $x_{\mathrm{Mn}}$ & $z_{\mathrm{Mn}}$ & $x_{\text {Ir }}$ & $z_{\mathrm{Ir}}$ & $x_{\mathrm{Si}}$ & $z_{\mathrm{Si}}$ \\
\hline LSDA & 155.918 & 0.6585 & 1.2376 & 0.0341 & 0.1858 & 0.1634 & 0.5627 & 0.7515 & 0.6201 \\
\hline $\begin{array}{l}\mathrm{LSDA}+U \\
(U=2 \mathrm{eV})\end{array}$ & 161.727 & 0.6470 & 1.2160 & 0.0331 & 0.1840 & 0.1648 & 0.5632 & 0.7622 & 0.6219 \\
\hline $\begin{array}{l}\mathrm{LSDA}+U \\
(U=3 \mathrm{eV})\end{array}$ & 165.693 & 0.6350 & 1.1869 & 0.0297 & 0.1817 & 0.1639 & 0.5618 & 0.7712 & 0.6244 \\
\hline $\begin{array}{l}\mathrm{LSDA}+U \\
(U=4 \mathrm{eV})\end{array}$ & 168.860 & 0.6268 & 1.1606 & 0.0266 & 0.1791 & 0.1625 & 0.5604 & 0.7771 & 0.6258 \\
\hline $\begin{array}{c}\text { Exp } \\
(10 \mathrm{~K})\end{array}$ & $170.14(2)$ & $0.61323(7)$ & $1.1411(1)$ & $0.0258(9)$ & $0.1719(8)$ & $0.1600(4)$ & $0.5604(4)$ & $0.7795(9)$ & $0.6290(8)$ \\
\hline
\end{tabular}

maps and BCOOP curves. In the TiNiSi-type structure, these atoms form a three-dimensional net of hexagons, in which the observed distances between $\mathrm{Ir}$ and $\mathrm{Si}$ in $\mathrm{IrMnSi}$ (2.33-2.42 $\AA$ at room temperature) are of similar magnitude as those reported for, e.g., (Ho/Yb) IrSi (Ref. 30) and $R \mathrm{IrSi}$ $(R=\mathrm{Tb}-\mathrm{Er}) .^{31}$ The covalent bond character in the hexagonal network has previously been reported from extended Hückelband-structure calculations for TiNiSi (Ref. 32) as well as from LMTO-ASA band-structure calculations for the isostructural EuAgMg. ${ }^{33}$

A cycloidal magnetic structure was found below a transition temperature of $460 \mathrm{~K}$ for IrMnSi. The propagation vector is parallel to the $c$ axis, and the magnetic moments rotate around an axis in the $a b$ plane. Thus Mn atoms in sheets perpendicular to the $c$ axis (having the same $z$ coordinate) have parallel magnetic moments, with the smallest $\mathrm{Mn}-\mathrm{Mn}$ separation within the sheet $d_{3}=3.8275(3) \AA$ at $10 \mathrm{~K}$. In contrast, the two shortest $\mathrm{Mn}-\mathrm{Mn}$ distances $\left(d_{1}\right.$ and $\left.d_{2}\right)$ are found between atoms in adjacent sheets "up" and "down" the $c$ axis, respectively, and thus the magnetic moments are rotated relative to each other. Pairs of $\mathrm{Mn}$ atoms separated $d_{1}[3.124(9) \AA$ at $10 \mathrm{~K}]$ have almost antiparallel moments $\left(163^{\circ}\right.$ angle), whereas pairs separated $d_{2}[3.313(4) \AA$ at $10 \mathrm{~K}$ ] have almost perpendicular moments $\left(82^{\circ}\right.$ angle) (see Figs. 7 and 8). The propagation vector of IrMnSi, $\mathbf{q}=[0,0,0.4530(5)]$ at $10 \mathrm{~K}$, is close to $\mathbf{q}=\left(0,0, \frac{1}{2}\right)$, which would correspond to a commensurate magnetic structure with a doubling of the $c$ axis, as was previously reported for the isostructural and isoelectronic compound RhMnSi. ${ }^{14}$ The experimentally observed magnetic structure of IrMnSi is corroborated by the results from the theoretical calculations. Both in fully self-consistent calculations and in calculations based on the force theorem an energy minimum is observed for the propagation vector $q \sim 0.43$ and for the angles between the Mn moments inside the crystallographic unit cell that are almost identical to the experimental ones.

The observed magnetic moment in IrMnSi, 3.8 $\mu_{B}$ at $10 \mathrm{~K}$, is higher than previous reports of $\mathrm{Mn}$ moments in the above-mentioned TiNiSi-type $\operatorname{Mn} M^{\prime} X$ compounds $\left(M^{\prime}=\right.$ transition metal element and $X=\mathrm{Si}$ or $\left.\mathrm{Ge}\right)$, and similar to the reported value for the ferromagnetic $\mathrm{Fe}_{2} \mathrm{P}$-type compound $\mathrm{PdMnGe}\left(3.7 \mu_{B}\right.$ at $\left.4.2 \mathrm{~K}\right) .{ }^{34}$ A similar magnitude of the Mn magnetic moment is also reported for the antiferromagnetic TiNiSi-type compounds NdMnGe $\left[\mu_{\mathrm{Mn}}=3.73(10) \mu_{B}, \mu_{\mathrm{Nd}}=3.30(8) \mu_{B} \text { at } 2 \mathrm{~K}\right]^{29}$ and $\mathrm{YMnGe}$ $\left[\mu_{\mathrm{Mn}}=3.51(15) \mu_{B}\right.$ at $\left.2 \mathrm{~K}\right] .{ }^{28}$ Of these, YMnGe forms a collinear antiferromagnetic order, ${ }^{28}$ whereas $\mathrm{NdMnGe}$ at $2 \mathrm{~K}$ has a cycloidal magnetic structure. ${ }^{29}$ The theoretical calculations based on LSDA give somewhat lower Mn moments for IrMnSi $\left(3.1-3.3 \mu_{B}\right)$ than the experimentally observed ones. If instead the $\mathrm{LSDA}+U$ approximation is used a perfect agreement with experimental values is observed, with calculated moments of 3.7-4.1 $\mu_{B}$ for $U$ values of $2-4 \mathrm{eV}$. These $U$ values are in good agreement with those previously discussed for metallic $3 d$ elements. ${ }^{24}$

The energy-band structure along the $-\mathrm{Z}-\Gamma-\mathrm{Z}$ direction calculated for different types of magnetic orders (see Fig. 12) shows a reduced number of bands crossing $E_{F}$ for the experimentally observed, noncollinear magnetic structure compared to those for a ferromagnet. Thus we conclude that IrMnSi belongs to the class of materials for which noncollinear magnetism is stabilized by the hybridization of energy bands opening band gaps around $E_{F}$, as discussed by Kübler ${ }^{3}$ and Lizárraga et al. ${ }^{5}$

\section{SUMMARY}

We reported a new phase IrMnSi that crystallizes with the TiNiSi-type structure. Below the magnetic transition temperature $460 \mathrm{~K}$ a cycloidal magnetic structure is formed, with magnetic moments on the Mn atoms of $3.8 \mu_{B}$ at $10 \mathrm{~K}$. The propagation vector is $\mathbf{q}=[0,0,0.4530(5)]$ at $10 \mathrm{~K}$, and additional phase angles $\left(\psi=0 ; \psi= \pm \frac{1}{2} q_{z}\right)$ relate the magnetic moments within the crystallographic cell. We found a certain covalency of the Ir-Si chemical bond, but an overall metallic character of the compound. The magnetic moments calculated with the LSDA are lower than the experimental values, whereas an agreement is reached when using the LSDA $+U$ approximation, with $U=2-4 \mathrm{eV}$. The experimentally observed magnetic structure is calculated to have the lowest energy, with good agreement between the observed and calculated lengths of the propagation vector as well as the angles between the magnetic moments within the crystallographic cell. A band mechanism is proposed to stabilize this noncollinear magnetic structure. 


\section{ACKNOWLEDGMENTS}

We are grateful to Juan Rodríguez-Carvajal for helpful comments on the use of the FULLPROF program for magnetic structure refinements and to John M. Wills for supplying the FP-LMTO code. Håkan Rundlöf is acknowledged for skillful assistance in neutron powder diffraction data collection. Financial support from the Swedish Research Council (VR) and the Swedish Foundation for Strategic Research (SSF) is acknowledged. O.E. also acknowledges support from the Göran Gustafsson foundation and the Center for Dynamical Processes, Uppsala.
${ }^{1}$ L. M. Sandratskii, Adv. Phys. 47, 91 (1998).

${ }^{2}$ J. Jensen and A. R. Macintosh, Rare-Earth Magnetism (Oxford University Press, Oxford, 1991).

${ }^{3}$ J. Kübler, Theory of Itinerant Electron Magnetism (Oxford Science Publications, Oxford, 2000).

${ }^{4}$ R. J. Elliot and E. A. Wedgewood, Proc. Phys. Soc. London 81, 846 (1963).

${ }^{5}$ R. Lizárraga, L. Nordström, L. Bergqvist, A. Bergman, E. Sjöstedt, P. Mohn, and O. Eriksson, Phys. Rev. Lett. 93, 107205 (2004).

${ }^{6}$ T. Eriksson, R. Lizárraga, S. Felton, L. Bergqvist, Y. Andersson, P. Nordblad, and O. Eriksson, Phys. Rev. B 69, 054422 (2004).

${ }^{7}$ T. Eriksson, L. Bergqvist, P. Nordblad, O. Eriksson, and Y. Andersson, J. Solid State Chem. 177, 4058 (2004).

${ }^{8}$ C. B. Shoemaker and D. P. Shoemaker, Acta Crystallogr. 18, 900 (1965).

${ }^{9}$ S. Nizioł, A. Bombik, W. Bażela, A. Szytuła, and D. Fruchart, J. Magn. Magn. Mater. 27, 281 (1982).

${ }^{10}$ W. Bażela, A. Szytuła, J. Todorović, and A. Zięba, Phys. Status Solidi A 64, 367 (1981).

${ }^{11}$ S. Nizioł, H. Bińczycka, A. Szytuła, J. Todorović, R. Fruchart, J. P. Senateur, and D. Fruchart, Phys. Status Solidi A 45, 591 (1978).

${ }^{12}$ H. Bińczycka, A. Szytuła, J. Todorović, T. Zaleski, and A. Zięba, Phys. Status Solidi A 35, K69 (1976).

${ }^{13}$ H. Fjellvåg and A. F. Andresen, J. Magn. Magn. Mater. 50, 291 (1985).

${ }^{14}$ W. Bażela-Wróbel, A. Szytuła, and J. Leciejewicz, Phys. Status Solidi A 82, 195 (1984).

${ }^{15}$ S. Rundqvist, Chem. Scr. 28, 15 (1988).

${ }^{16}$ J. Rodríguez-Carvajal, FULLPROF computer program, version 2.80 (LLB, Saclay, 2004).

${ }^{17}$ Components along the crystallographic axes: $m_{x}$ $=M \sin \theta \cos \varphi, m_{y}=M \sin \theta \sin \varphi, m_{z}=M \cos \theta$.

${ }^{18}$ J. M. Wills, O. Eriksson, M. Alouani, and D. L. Price, in Electronic Structure and Physical Properties of Solids, edited by $\mathrm{H}$.
Dreyssé (Springer, Berlin, 2000).

${ }^{19}$ A. Grechnev, R. Ahuja, and O. Eriksson, J. Phys.: Condens. Matter 15, 7751 (2003).

${ }^{20}$ The computer code was made in house, based on the original method published in O. K. Andersen, Phys. Rev. B 12, 3060 (1975) and H. L. Skriver, The LMTO Method (Springer, Berlin, 1984).

${ }^{21}$ G. Kresse and J. Hafner, Phys. Rev. B 47, 558 (1993).

${ }^{22}$ V. I. Anisimov, J. Zaanen, and O. K. Andersen, Phys. Rev. B 44, 943 (1991).

${ }^{23}$ For technical reasons this calculation was made not in a cycloidal geometry, but in a screw-spiral geometry. However, the angles between all atoms in the theoretical work was identical to those reported in the experiment.

${ }^{24}$ L. Chioncel, M. I. Katsnelson, R. A. de Groot, and A. I. Lichtenstein, Phys. Rev. B 68, 144425 (2003).

${ }^{25}$ W. Bażela, A. Szytuła, J. Todorović, Z. Tomkovicz, and A. Zięba, Phys. Status Solidi A 38, 721 (1976).

${ }^{26}$ V. Johnson, Inorg. Chem. 14, 1117 (1975).

${ }^{27}$ J. Ijjaali, R. Welter, G. Venturini, and B. Malaman, J. Alloys Compd. 292, 4 (1999).

${ }^{28}$ V. Klosek, A. Vernière, B. Ouladdiaf, and B. Malaman, J. Magn. Magn. Mater. 256, 69 (2003).

${ }^{29}$ R. Welter, G. Venturini, I. Ijjaali, and B. Malaman, J. Magn. Magn. Mater. 205, 221 (1999).

${ }^{30}$ R. Mishra, R-D. Hoffmann, and R. Pöttgen, Z. Anorg. Allg. Chem. 627, 1787 (2001).

${ }^{31}$ A. Szytuła, M. Hoffmann, J. Leciejewicz, B. Penc, and A. Zygmunt, J. Alloys Compd. 316, 58 (2001).

${ }^{32}$ G. A. Landrum, R. Hoffmann, J. Evers, and H. Boysen, Inorg. Chem. 37, 5754 (1998).

${ }^{33}$ D. Johrendt, G. Kotzyba, H. Trill, B. D. Mosel, H. Eckert, T. Fickenscher, and R. Pöttgen, J. Solid State Chem. 164, 201 (2002).

${ }^{34}$ A. Vernière, J. Tobola, G. Venturini, and B. Malaman, J. Magn. Magn. Mater. 207, 95 (1999). 